\title{
Role of Multiple Scattering in Cross-Correlated Light Scattering with a Single Laser Beam
}

James A. Lock

Cleveland State University, j.lock@csuohio.edu

Follow this and additional works at: https://engagedscholarship.csuohio.edu/sciphysics_facpub

Part of the Physics Commons

How does access to this work benefit you? Let us know!

\section{Publisher's Statement}

This paper was published in Applied Optics and is made available as an electronic reprint with the permission of OSA. The paper can be found at the following URL on the OSA website: http://www.opticsinfobase.org/ao/abstract.cfm?URI=ao-36-30-7559. Systematic or multiple reproduction or distribution to multiple locations via electronic or other means is prohibited and is subject to penalties under law.

\section{Original Citation}

Lock, James A. "Role of Multiple Scattering in Cross-Correlated Light Scattering with a Single Laser Beam." Applied Optics 36 (1997): 7559-7570.

\section{Repository Citation}

Lock, James A., "Role of Multiple Scattering in Cross-Correlated Light Scattering with a Single Laser Beam" (1997). Physics Faculty Publications. 105.

https://engagedscholarship.csuohio.edu/sciphysics_facpub/105

This Article is brought to you for free and open access by the Physics Department at EngagedScholarship@CSU. It has been accepted for inclusion in Physics Faculty Publications by an authorized administrator of EngagedScholarship@CSU. For more information, please contact library.es@csuohio.edu. 


\title{
Role of multiple scattering in cross-correlated light scattering with a single laser beam
}

\author{
James A. Lock
}

\begin{abstract}
Previous systems for measuring cross-correlated light scattering by small particles suspended in a liquid with multiple-scattering suppression have illuminated the particles with two laser beams. It is shown that multiple-scattering suppression should also occur in cross correlation for a system that employs a single laser beam and two closely spaced detectors with wide fields of view. The single-scattering, doublescattering, and single-double-scattering cross-term contributions to the intensity cross-correlation function are calculated. It is found that the two cross terms, when added together, are unimportant for both autocorrelation and cross correlation. The amplitude of the double-scattering term can be greatly diminished by judicious detector spacing because the spatial coherence area in the detector plane for double scattering is much smaller than that for single scattering. () 1997 Optical Society of America
\end{abstract}

\section{Introduction}

Over the past three decades, dynamic light scattering has become the nonintrusive measurement technique of choice for studying small particles suspended in a liquid. Single scattering dominates if the suspension is dilute. In this case the autocorrelation function of the light scattered by noninteracting particles decays exponentially with time, and the particle diffusion coefficient is proportional to the decay rate. ${ }^{1}$ Multiple scattering is dominant in dense suspensions, and its presence complicates the time dependence of the autocorrelation function. This complication has been addressed in three ways. For semidilute systems in which multiple scattering first becomes significant, various investigators have calculated and compensated for the effects of double ${ }^{2-5}$ and multiple $e^{6,7}$ scattering. For dense suspensions in the deep multiplescattering regime, others have modeled multiple scattering by using photon diffusion ${ }^{8-11}$ and radiative transfer ${ }^{12,13}$ methods. Last, others have employed cross correlation by using two laser beams to suppress the contribution of multiple scattering to the cross-correlation function relative to that of single scattering. ${ }^{14-20}$

In 1981, Phillies proposed a scattering geometry

The author is with the Department of Physics, Cleveland State University, Cleveland, Ohio 44115.

Received 19 February 1997; revised manuscript received 19 May 1997.

0003-6935/97/307559-16\$10.00/0

(C) 1997 Optical Society of America that uses two counterpropagating laser beams that have the same wavelength and two oppositely positioned detectors, and he predicted that single scattering would be strongly cross correlated whereas multiple scattering would be uncorrelated. ${ }^{14}$ Subsequent experiments verified that the expected multiplescattering suppression occurred.15,16 A few years later, the complete cross-correlation function for the two-beam system was calculated, ${ }^{17}$ and a refinement that uses two laser beams of different wavelengths with filters in front of the detectors was proposed in order to separate the contributions to the crosscorrelation function from scattering at angles $\theta$ and $\pi-\theta$. Again, subsequent experiments verified that the expected multiple-scattering suppression occurred for the two-wavelength cross-correlation system. ${ }^{18-20}$

A common concern for these cross-correlation systems is that they are difficult to align. Thus there has been motivation to find simpler scattering geometries that suppress multiple scattering in cross correlation. The use of monomode optical fibers as detectors ${ }^{21-25}$ has opened up new possibilities in this regard, and an autocorrelation backscatter probe operating in the scattering near zone that suppresses multiple scattering has been developed. ${ }^{26}$

Here I present a theoretical analysis of a recently proposed $^{27}$ cross-correlation geometry that has been found to suppress multiple scattering in dense suspensions and that is easier to align than the twobeam cross-correlation systems. The system that we study here consists of a single laser beam and two closely spaced detectors with wide fields of view. ${ }^{27-29}$ It is shown that multiply scattered light should cease 
being cross correlated for small detector spacings, whereas singly scattered light ceases being cross correlated for larger detector spacings. Thus if two monomode optical fibers coupled to detectors are positioned beyond the multiple-scattering threshold separation but lie within the single-scattering threshold separation, the multiple-scattering contribution to the cross-correlation function should be strongly suppressed with respect to the singlescattering contribution. A preliminary version of this calculation that ignores both the polarization and the angular dependence of the scattered light has already appeared..$^{30}$

There are many geometries for which multiple scattering is suppressed in autocorrelation or cross correlation, and the method examined here is only one of them. Another possibility that has been suggested informally by a number of researchers is to perform autocorrelation with the field of view of a collimated detector centered on a point in the laser beam in the scattering cell. At this location the singly scattered light is bright while the multiply scattered light along the detector field of view is much dimmer, again producing multiple-scattering suppression relative to single scattering. Further experimentation will determine which of the many feasible techniques is most efficient at suppressing multiple scattering and which is the easiest to use. The purpose here is more modest, namely, to provide a theoretical analysis of the one-beam cross-correlation technique.

The body of this paper is organized as follows. In Section 2 the scattering geometry is described and the notation is defined. In Sections 3 and 4 single and double scattering, respectively, by a collection of noninteracting spherical particles suspended in a liquid are considered. In Section 5 the two electricfield correlation functions are calculated for the onebeam cross-correlation system. The dominant cross-correlation function includes geometric factors related to the far-zone spatial coherence areas for single and double scattering. In Section 6 the intensity cross-correlation function is calculated, the degree of double-scattering suppression is determined, and the time dependences of the autocorrelation and cross-correlation functions are determined. Finally, in Section 7, results are discussed. In suspensions for which a substantial amount of double scattering occurs, higher orders of multiple scattering are usually important as well. Here, the calculations are limited to double scattering, however, because the spatial coherence area argument for doublescattering suppression can be straightforwardly generalized to higher orders of multiple scattering.

\section{Scattering Geometry}

We follow the general procedure used to analyze twobeam cross-correlation systems in Refs. 17 and 20 . The scattering geometry is illustrated in Fig. 1. A cell of volume $V$ and with transparent walls contains $N$ monodisperse nonabsorbing dielectric spherical particles of real refractive index $n_{p}$ and radius $a$ suspended in a nonabsorbing liquid of real refractive index $n_{L}$.

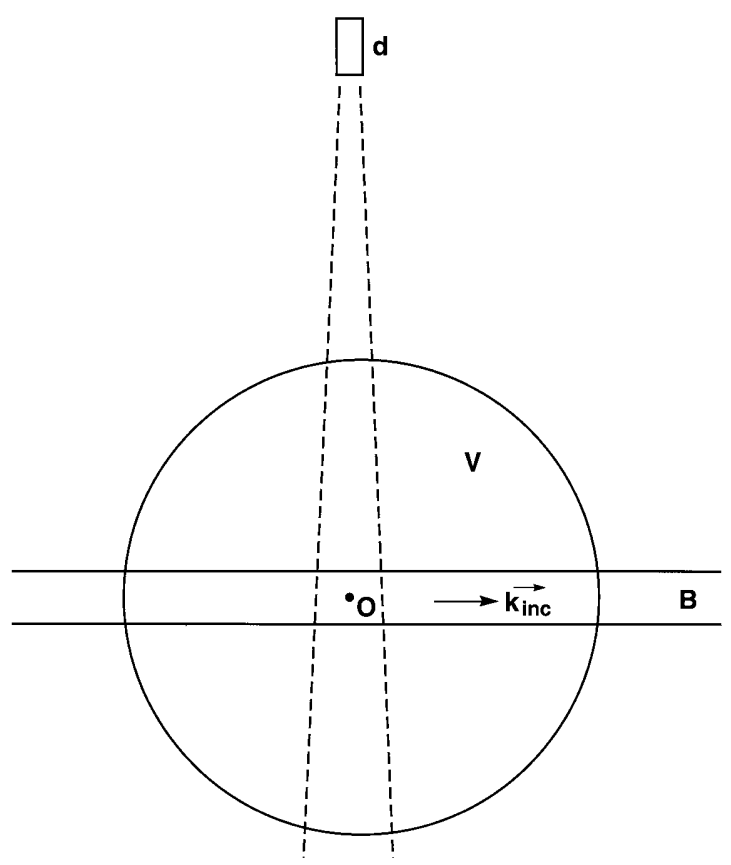

Fig. 1. Focused laser beam $B$ that has the wave vector $\mathbf{k}_{\text {inc }}$ propagates through a scattering cell of volume $V$, which contains $N$ particles suspended in a liquid. The field of view of detector $d$ is given by the dashed lines. The origin of coordinates $O$ is at the center of the intersection volume of the beam and the detector field of view.

The average number density of the suspended particles is $\rho=N / V$, their volume fraction is $\phi=4 \pi a^{3} \rho / 3$, and their refractive index relative to the liquid is $n=$ $n_{p} / n_{L}$. A monochromatic laser beam of wavelength $\lambda$ in vacuum and angular frequency $\omega$ propagates through the cell and is scattered by the particles. The electric field of the unscattered portion of the beam at the position $\mathbf{r}_{j}$ in the cell at time $t_{j}$ is

$$
\begin{aligned}
\mathbf{E}_{\text {inc }}\left(\mathbf{r}_{j}, t_{j}\right)= & E_{0} B\left(\mathbf{r}_{j}\right) \exp \left(-L_{b j} / 2 L_{\text {scatt }}\right) \hat{u}_{\text {inc }} \\
& \times \exp \left(i \mathbf{k}_{\text {inc }} \cdot \mathbf{r}_{j}-i \omega t_{j}\right),
\end{aligned}
$$

where $E_{0}$ is the incident field strength, $B\left(\mathbf{r}_{j}\right)$ is a dimensionless function describing the transverse beam profile, $L_{b j}$ is the distance the beam has traveled through the suspension to the position $\mathbf{r}_{j}, L_{\text {scatt }}$ is the single-scattering mean free path, ${ }^{31} \hat{u}_{\text {inc }}$ is the beam-polarization direction, and the wave number of the light in the liquid is

$$
k \equiv\left|\mathbf{k}_{\text {inc }}\right|=\frac{2 \pi n_{L}}{\lambda}
$$

The beam is scattered by particle $j$ at position $\mathbf{r}_{j}$ at time $t_{j}$. The scattered electric field at the far-zone position $\mathbf{R}$ in the cell at later time $T$ is

$$
\begin{aligned}
\mathbf{E}_{\text {scatt }}(\mathbf{R}, T)= & \frac{E_{0} B\left(\mathbf{r}_{j}\right) \mathbf{A}_{\text {Mie }}}{k R} \\
& \times \exp \left[-\left(L_{b j}+L_{j R}\right) / 2 L_{\text {scatt }}\right] \\
& \times \exp \left[i k R-i \omega T-i \mathbf{\kappa} \cdot \mathbf{r}_{j}\left(t_{j}\right)\right],
\end{aligned}
$$




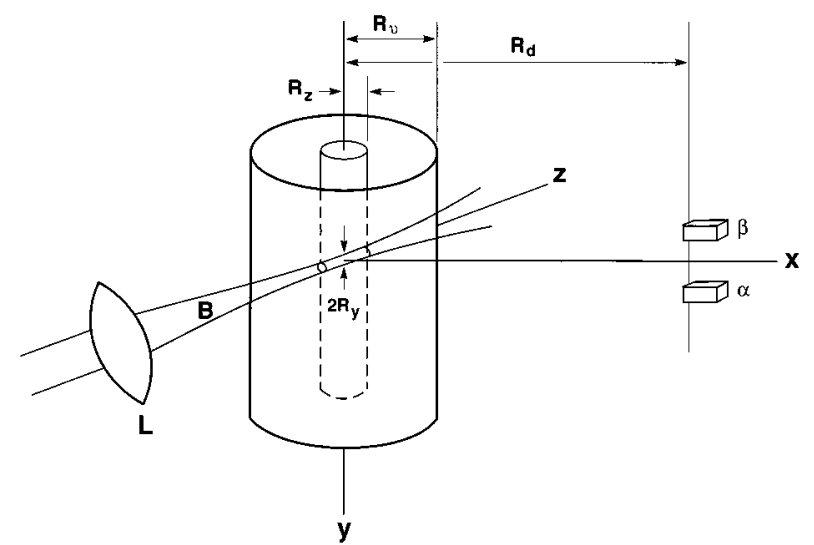

Fig. 2. Laser beam B is focused by lens $\mathrm{L}$ to half-width $R_{y}$. It propagates through a test tube of radius $R_{z}$, which contains the liquid and the suspended particles. The test tube is enclosed in a coaxial index-matching vat of radius $R_{v}$. Wide-field-of-view detectors $\alpha$ and $\beta$ with completely overlapping fields of view in the scattering cell are a distance $R_{d}$ from the center of the vat.

where the distance in the suspension from particle $j$ to position $\mathbf{R}$ is $L_{j R}$, the scattered wave vector $\mathbf{k}_{\text {scatt }}$ is

$$
\mathbf{k}_{\text {scatt }}=\frac{k \mathbf{R}}{R}
$$

the scattered momentum transfer $\boldsymbol{\kappa}$ is

$$
\begin{aligned}
\mathbf{\kappa} & =\mathbf{k}_{\text {scatt }}-\mathbf{k}_{\text {inc }}, \\
|\boldsymbol{\kappa}| & =2 k \sin (\theta / 2),
\end{aligned}
$$

$\theta$ is the scattering angle, and $\mathbf{A}_{\mathrm{Mie}}$ is the far-zone Lorenz-Mie scattering amplitude. For the special case of Rayleigh scattering, ${ }^{32}$ i.e., $k a \ll 1$, we have

$$
\begin{aligned}
\lim _{k a \ll 1} \mathbf{A}_{\text {Mie }} & =\mathbf{A}_{\text {Rayleigh }} \\
& =\left(\frac{n^{2}-1}{n^{2}+2}\right)(k a)^{3} \hat{k}_{\text {scatt }} \times\left(\hat{u}_{\text {inc }} \times \hat{k}_{\text {scatt }}\right) .
\end{aligned}
$$

We consider the case in which the wave vector of the incident laser beam is in the positive $z$ direction. We also assume that the scattering cell is a thin-wall cylindrical test tube with its symmetry axis along the $y$ axis, as shown in Fig. 2. It is surrounded by a coaxial cylindrical vat filled with the same liquid as is in the test tube, corresponding to the experimental geometry of Refs. 27-29. A single-point detector $\mathrm{d}$ is placed at the position $\mathbf{R}=\mathbf{R}_{d}$ in the $x z$ plane for autocorrelation, or a pair of them, $\alpha$ and $\beta$, are placed slightly above and below the $x z$ plane for cross correlation, so that

$$
\hat{k}_{\text {scatt }} \approx \sin \theta \hat{u}_{x}+\cos \theta \hat{u}_{z} .
$$

The four input-output polarization channels for this geometry are denoted by $V V, V H, H V$, and $H H$ and are illustrated in Fig. 3. For single scattering in the $x z$ plane, we can rewrite Eq. (6) for all four polarization channels by using the compact notation

$$
\left\langle\pi_{f}\left|\mathbf{A}_{\text {Rayleigh }}\right| \pi_{i}\right\rangle=A_{1}\left[P_{1}\right],
$$

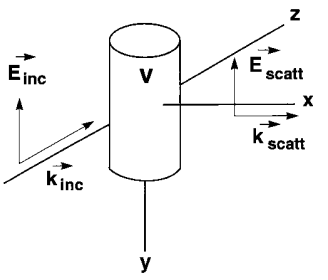

(a)

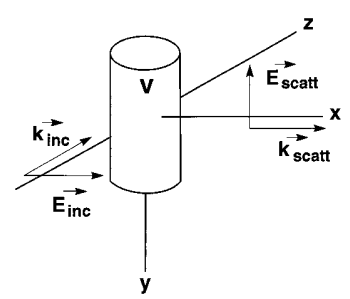

(c) (b)

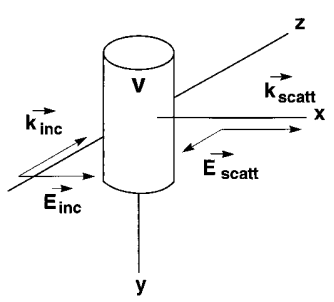

(d)

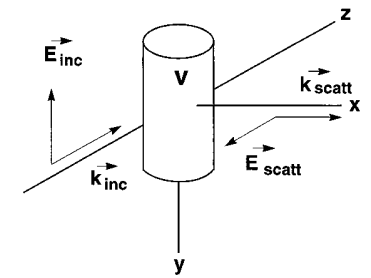

Fig. 3. Four input-output polarization states for scattering in the xz plane: (a) $V V$, (b) $V H$, (c) $H V$, (d) $H H$.

where $\pi_{i}$ is the initial polarization, $\pi_{f}$ is the final polarization (each $V$ or $H$ ),

$$
\begin{aligned}
A_{1} & =(k a)^{3}\left(n^{2}-1\right) /\left(n^{2}+2\right), \\
{\left[P_{1}\right] } & =\left[\begin{array}{ll}
V V_{1} & V H_{1} \\
H V_{1} & H H_{1}
\end{array}\right] .
\end{aligned}
$$

In Eqs. (8)-(10) the subscript 1 denotes single scattering. The angular dependence of the elements of $\left[P_{1}\right]$ is

$$
\begin{aligned}
V V_{1} & =1, \\
V H_{1} & =H V_{1}=0, \\
H H_{1} & =\cos \theta .
\end{aligned}
$$

For double scattering by particles $j$ and then $l$, the scattered field of particle $j$ is the incident field for particle $l$. Iterating Eq. (6), assuming that $l$ is in the far zone of $j$, we can write the Rayleigh amplitude for double scattering for all four polarization channels with the detector in the $x z$ plane by using the compact notation $A_{2}\left[P_{2}\right]$, where

$$
\begin{aligned}
A_{2} & =A_{1}^{2}, \\
{\left[P_{2}\right] } & =\left[\begin{array}{ll}
V V_{2} & V H_{2} \\
H V_{2} & H H_{2}
\end{array}\right],
\end{aligned}
$$

with

$$
\begin{aligned}
V V_{2}= & 1-\sin ^{2} \theta_{j l} \sin ^{2} \phi_{j l}, \\
V H_{2}= & \sin ^{2} \theta_{j l} \sin \phi_{j l} \cos \phi_{j l} \cos \theta \\
& -\sin \theta_{j l} \cos \theta_{j l} \sin \phi_{j l} \sin \theta \\
H V_{2}= & -\sin ^{2} \theta_{j l} \sin \phi_{j l} \cos \phi_{j l}, \\
H H_{2}= & \cos \theta-\sin ^{2} \theta_{j l} \cos ^{2} \phi_{j l} \cos \theta \\
& +\sin \theta_{j l} \cos \theta_{j l} \cos \phi_{j l} \sin \theta .
\end{aligned}
$$


The intermediate wave vector $\mathbf{k}_{j l}$ of the light scattered by $j$ and incident upon $l$ is

$$
\mathbf{k}_{j l}=k\left(\sin \theta_{j l} \cos \phi_{j l} \hat{u}_{x}+\sin \theta_{j l} \sin \phi_{j l} \hat{u}_{y}+\cos \theta_{j l} \hat{u}_{z}\right) .
$$

For the remainder of this paper, all quantities in brackets are to be evaluated for all four input-output polarization channels.

Finally, the relative sensitivity ${ }^{17}$ of detector $d$ to light that has its last interaction with a particle at position $\mathbf{r}$ is $D(\mathbf{r})$. Only some of the particles in the cell are illuminated by the laser beam and only some of these in turn are in the field of view of the detector. The volume of the laser beam within the cell, the volume of the detector field of view within the cell, and the intersection of these two volumes are denoted by $V_{1}, V_{2}$, and $V_{0}$, respectively; the average numbers of particles in these volumes are $N_{1}, N_{2}$, and $N_{0}$. The origin of the coordinates is taken to be at the center of $V_{0}$, as in Fig. 1.

\section{Single Scattering by a Collection of Particles}

We now consider light that reaches detector $d$ at time $T$ because of single scattering of the laser beam by the suspended particles at positions $\mathbf{r}_{j}$ in the cell at earlier times $t_{j}$, as is illustrated in Fig. 4(a). Because the participating particles are both illuminated by the laser beam and in the detector field of view, the single-scattered electric field at the detector is

$$
\begin{aligned}
{\left[E_{d 1}\left(\mathbf{R}_{d}, T\right)\right]=} & \frac{E_{0} A_{1}\left[P_{1}\right]}{k R_{d}} \exp \left(i k R_{d}-i \omega T\right) \sum_{j=1}^{N_{0}} B\left(\mathbf{r}_{j}\right) D\left(\mathbf{r}_{j}\right) \\
& \times \exp \left[-\left(L_{b j}+L_{j d}\right) / 2 L_{\text {scatt }}\right] \\
& \times \exp \left[-i \boldsymbol{\kappa} \cdot \mathbf{r}_{j}\left(t_{j}\right)\right],
\end{aligned}
$$

where the subscripts $d$ and 1 label the detector and denote single scattering, respectively. In obtaining Eq. (16) we assume that the incident laser beam was focused by a lens upbeam from the cell, as in Fig. 2, so that the cell lies entirely within the beam focal waist. As a result, $\mathbf{k}_{\text {inc }}$ is independent of $\mathbf{r}_{j}$. We also assume that the point detector for autocorrelation or the pair of point detectors for cross correlation are placed along the $x$ axis and have wide fields of view, i.e., the radius of a detector field of view in the plane containing the laser beam is much wider than the beam radius. Such detectors have not often been considered because of concerns that they record a large range of $\boldsymbol{\kappa}$ values, causing instrumental broadening. Only a singlescattered momentum transfer is recorded, and instrumental broadening is thereby avoided, however, if either bare-end monomode optical fibers coupled to detectors are placed on the paraxial focal line of the index-matching vat ${ }^{27,28}$ or if gradient-index lenses are cemented onto the end of the monomode fibers placed far from the vat.29 For these two geometries, all singly scattered light reaching the detectors has the same scattering angle (i.e., $\theta=90^{\circ}$ ) and thus the same scattered momentum transfer.

The noninteracting suspended particles in the scat-

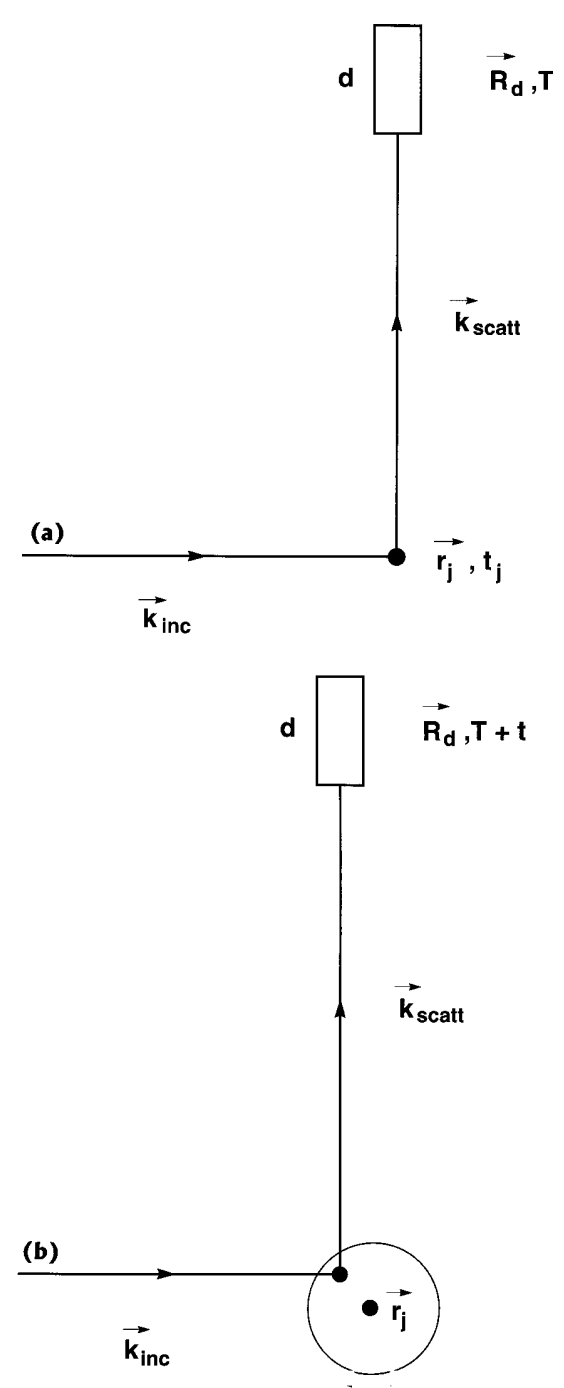

Fig. 4. Single scattering by particle $j$ (a) at time $t_{j}$ when the particle is at position $\mathbf{r}_{j}$, (b) at time $\tau$ later when the particle has moved by means of diffusion to somewhere within a sphere of radius $\Delta_{\text {ave }}(\tau)$ centered on $\mathbf{r}_{j}$.

tering cell are in random motion. The probability that particle $j$ is at position

$$
\mathbf{r}_{j}\left(t_{j}+\tau\right)=\mathbf{r}_{j}\left(t_{j}\right)+\Delta_{j}(\tau)
$$

at time $t_{j}+\tau$, given that it was at position $\mathbf{r}_{j}$ at the earlier time $t_{j}$, is $P\left(\Delta_{j}, \tau\right)$. The singly scattered electric field reaching the detector at $T+\tau$ averaged over all the possible particle displacements $\boldsymbol{\Delta}_{j}$ at $t_{j}+\tau$, as in Fig. 4(b), is then

$$
\begin{aligned}
{\left[E_{d 1}\left(\mathbf{R}_{d}, T+\tau\right)\right]=} & \frac{E_{0} A_{1}\left[P_{1}\right]}{k R_{d}} \\
& \times \exp [i k R-i \omega(T+\tau)] S^{*}(\boldsymbol{\kappa}, \tau) \\
& \times \sum_{j=1}^{N_{0}} B\left(\mathbf{r}_{j}\right) D\left(\mathbf{r}_{j}\right) \\
& \times \exp \left[-\left(L_{b j}+L_{j d}\right) / 2 L_{\text {scatt }}\right] \\
& \times \exp \left[-i \kappa \cdot \mathbf{r}_{j}\left(t_{j}\right)\right] .
\end{aligned}
$$


In Eq. (18) we assume that the same $N_{0}$ particles are within $V_{0}$ at times $t_{j}$ and $t_{j}+\tau$ and that both the beam profile and the detector field of view are sufficiently slowly spatially varying and $\tau$ is sufficiently small so that

$$
\begin{aligned}
B\left[\mathbf{r}_{j}\left(t_{j}\right)\right] & \approx B\left[\mathbf{r}_{j}\left(t_{j}+\tau\right)\right], \\
D\left[\mathbf{r}_{j}\left(t_{j}\right)\right] & \approx D\left[\mathbf{r}_{j}\left(t_{j}+\tau\right)\right] .
\end{aligned}
$$

The structure factor of the particles' random motion is

$$
S(\boldsymbol{\kappa}, \tau) \equiv \int d^{3} \Delta_{j} P\left(\boldsymbol{\Delta}_{j}, \tau\right) \exp \left(i \boldsymbol{\kappa} \cdot \boldsymbol{\Delta}_{j}\right)
$$

\section{Double Scattering by a Collection of Particles}

We consider the double-scattering situation of Fig. 5(a). Light scatters from particle $j$ at position $\mathbf{r}_{j}$ at time $t_{j}$ and travels the distance

$$
r_{j l}=\left|\mathbf{r}_{j}\left(t_{j}\right)-\mathbf{r}_{l}\left(t_{l}\right)\right|
$$

without further interaction to position $\mathbf{r}_{l}$ at time $t_{l}$, where it scatters from particle $l$. The doubly scattered light then propagates to the detector in the far zone without further interaction with the particles. For this situation, particle $j$ is illuminated by the incident beam, and particle $l$ is in the field of view of the detector. We assume that particle $l$ is also in the scattering far zone of particle $j$. The far-zone assumption is reasonable for semidilute suspensions because the largest near-zone contribution to the scattered electric field [i.e., the term proportional to $\left.\left(k r_{j l}\right)^{-2}\right]$ for Rayleigh scattering with ${ }^{27,28} a=0.053$ $\mu \mathrm{m}$ is less than 0.1 of the far-zone contribution [i.e., the term proportional to $\left.\left(k r_{i l}\right)^{-1}\right]$ for $\phi \leq 0.01$. The doubly scattered electric field reaching the detector at time $T$ is then

$$
\begin{aligned}
{\left[E_{2 d}\left(\mathbf{R}_{d}, T\right)\right]=} & \frac{E_{0} A_{2}}{k R_{d}} \exp \left(i k R_{d}-i \omega T\right) \\
& \times \sum_{j=1}^{N_{1}} \sum_{l=1}^{N_{2}}\left[P_{2}\right] \frac{B\left(\mathbf{r}_{j}\right) D\left(\mathbf{r}_{l}\right)}{k r_{j l}} \\
& \times \exp \left[-\left(L_{b j}+r_{j l}+L_{l d}\right) / 2 L_{\text {scatt }}\right] \\
& \times \exp \left[i\left(\mathbf{k}_{\text {inc }}-\mathbf{k}_{j l}\right) \cdot \mathbf{r}_{j}\left(t_{j}\right)\right] \\
& \times \exp \left[i\left(\mathbf{k}_{j l}-\mathbf{k}_{\text {scatt }}\right) \cdot \mathbf{r}_{l}\left(t_{l}\right)\right]
\end{aligned}
$$

For light reaching the detector at later time $T+\tau$, as in Fig. 5(b), we further assume that $\Delta_{j}, \Delta_{l} \ll r_{j l}$ so that the intermediate wave vector from any of the possible positions of the first scattering to any of the possible positions of the second scattering is independent of $\boldsymbol{\Delta}_{j}$ and $\boldsymbol{\Delta}_{l}$. The doubly scattered electric field reaching the detector at $T+\tau$ averaged over all the
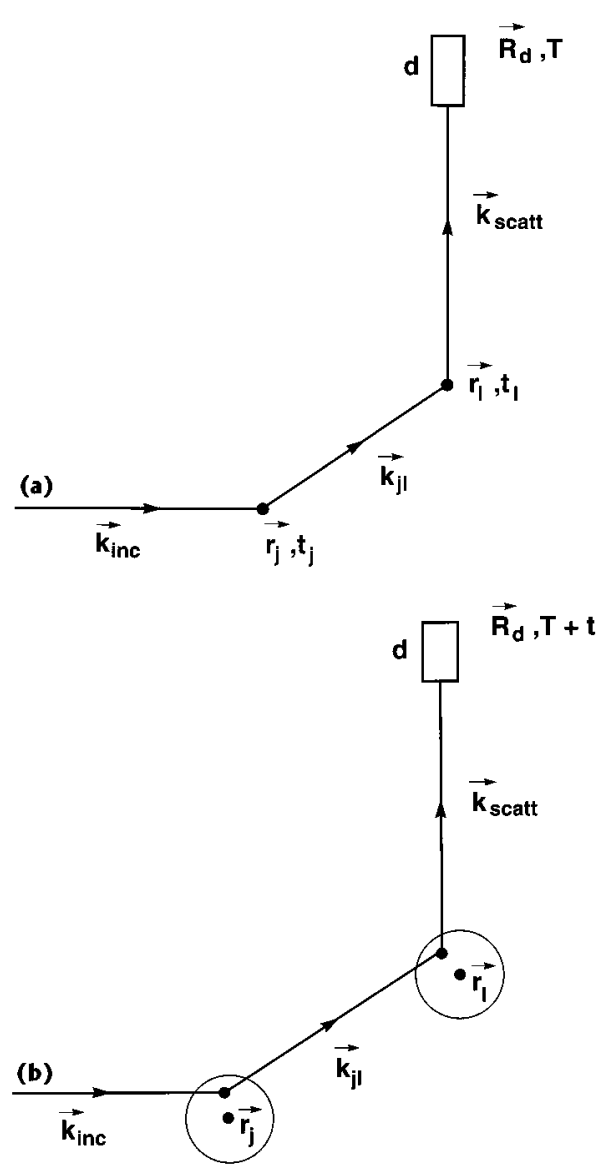

Fig. 5. Double scattering by particles $j$ and $l$ (a) at times $t_{j}$ and $t_{l}$ when the particles are at the positions $\mathbf{r}_{j}$ and $\mathbf{r}_{l}$, (b) at time $\tau$ later when the particles have moved by means of diffusion to somewhere within spheres of radii $\Delta_{\text {ave }}(\tau)$ centered on $\mathbf{r}_{j}$ and $\mathbf{r}_{l}$.

possible particle displacements $\Delta_{j}$ and $\Delta_{l}$ at $t_{j}+\tau$ and $t_{l}+\tau$ is then

$$
\begin{aligned}
{\left[E_{2 d}\left(\mathbf{R}_{d}, T+\tau\right)\right]=} & \frac{E_{0} A_{2}}{k R_{d}} \exp \left[i k R_{d}-i \omega(T+\tau)\right] \\
& \times \sum_{j=1}^{N_{1}} \sum_{l=1}^{N_{2}}\left[P_{2}\right] \frac{B\left(\mathbf{r}_{j}\right) D\left(\mathbf{r}_{l}\right)}{k r_{j l}} \\
& \times \exp \left[-\left(L_{b j}+r_{j l}+L_{l d}\right) / 2 L_{\text {scatt }}\right] \\
& \times \exp \left[i\left(\mathbf{k}_{\text {inc }}-\mathbf{k}_{j l}\right) \cdot \mathbf{r}_{j}\left(t_{j}\right)\right] \\
& \times \exp \left[i\left(\mathbf{k}_{j l}-\mathbf{k}_{\text {scatt }}\right) \cdot \mathbf{r}_{l}\left(t_{l}\right)\right] \\
& \times S^{*}\left(\mathbf{k}_{\text {scatt }}-\mathbf{k}_{j l}, \tau\right) S^{*}\left(\mathbf{k}_{j l}-\mathbf{k}_{\text {inc }}, \tau\right) .
\end{aligned}
$$

\section{Electric-Field Correlation Functions}

In this section we consider two closely spaced detectors, $\alpha$ and $\beta$, in the scattering far zone, as in Fig. 2. The angle between the detectors is $\delta$, and their fields of view within the scattering cell almost completely overlap, i.e.,

$$
D_{\alpha}(\mathbf{r}) \approx D_{\beta}(\mathbf{r}) \equiv D(\mathbf{r})
$$


We consider the two electric-field cross-correlation functions,

$$
\begin{gathered}
{[c(\tau)]=\int_{-\infty}^{\infty} \mathrm{d} T\left[E_{\alpha} *(T+\tau) E_{\beta}(T)\right],} \\
{\left[c^{\prime}(\tau)\right]=\int_{-\infty}^{\infty} \mathrm{d} T\left[E_{\alpha}(T+\tau) E_{\beta}(T)\right],}
\end{gathered}
$$

where the electric field at each detector for each of the four input-output polarization channels is the sum of single- and double-scattering contributions. The correlation function $[c(\tau)]$ is dominant for autocorrelation $^{1}$ (i.e., $\delta=0$ ), and $\left[c^{\prime}(\tau)\right]$ is dominant for cross correlation for which two counterpropagating beams and two oppositely positioned detectors are used. ${ }^{17}$

The $T$ integral in Eqs. (25) and (26) is modeled by the ensemble average of the integrands $\left\langle\left[E_{\alpha}{ }^{*}(\tau) E_{\beta}(0)\right]\right\rangle$ and $\left\langle\left[E_{\alpha}(\tau) E_{\beta}(0)\right]\right\rangle$, respectively, i.e., the product of the fields is averaged over all the possible positions of particles $j$ and $l$ at times $t_{j}$ and $t_{l}$ and times $t_{j}+\tau$ and $t_{l}+$ $\tau$ when scattering occurs. ${ }^{14}$ Averaging over the particle positions at $t_{j}+\tau$ and $t_{l}+\tau$, given the positions at $t_{j}$ and $t_{l}$, was performed in Sections 3 and 4 and led to the scattering structure factors. We now average over the positions of the particles at $t_{j}$ and $t_{l}$. For the single-scattering contribution to the electric-field correlation function $[c(\tau)]$, substitution of Eqs. (16) and (18) into Eq. (25) gives ${ }^{33}$

$$
\begin{aligned}
{\left[c_{1}(\tau)\right] \approx } & \left\langle\left[E_{\alpha 1}{ }^{*}(\tau) E_{\beta 1}(0)\right]\right\rangle \\
= & \frac{E_{0}{ }^{2} A_{1}^{2}\left[P_{1}^{2}\right]}{k^{2} R_{d}^{2}} \exp (i \omega \tau) S\left(\boldsymbol{\kappa}^{\alpha}, \tau\right) \\
& \times\left\{\rho \int_{V_{0}} \mathrm{~d}^{3} r_{j}\left|B\left(\mathbf{r}_{j}\right) D\left(\mathbf{r}_{j}\right)\right|^{2}\right. \\
& \times \exp \left[-\left(L_{b j}+L_{j d}\right) / L_{\mathrm{scatt}}\right] \exp \left(i \boldsymbol{\epsilon} \cdot \mathbf{r}_{j}\right) \\
& +\rho^{2} \int_{V_{0}} \mathrm{~d}^{3} r_{j} \int_{V_{0}} \mathrm{~d}^{3} r_{j m} B^{*}\left(\mathbf{r}_{j}\right) D^{*}\left(\mathbf{r}_{j}\right) B\left(\mathbf{r}_{m}\right) D\left(\mathbf{r}_{m}\right) \\
& \times\left[g\left(r_{j m}\right)-1\right] \exp \left[-\left(L_{b j}+L_{j d}+L_{b m}+L_{m d}\right) / 2 L_{\text {scatt }}\right] \\
& \times \exp \left(i \in \cdot \mathbf{r}_{j}\right) \exp \left(-i \boldsymbol{\kappa}^{\beta} \cdot \mathbf{r}_{j m}\right) \\
& +\rho^{2} \mid \int_{V_{0}} d^{3} r_{j} B\left(\mathbf{r}_{j}\right) D\left(\mathbf{r}_{j}\right) \exp \left[-\left(L_{b j}+L_{j d}\right) / 2 L_{\text {scatt }}\right] \\
& \left.\times\left.\exp \left(-i \boldsymbol{\kappa}_{\text {ave }} \cdot \mathbf{r}_{j m}\right)\right|^{2}\right\},
\end{aligned}
$$

where the subscript 1 denotes single scattering, the elements of $\left[P_{1}^{2}\right]$ are the squares of the elements of $\left[P_{1}\right]$, and

$$
\begin{aligned}
\boldsymbol{\epsilon} & =\mathbf{k}_{\text {scatt }}^{\alpha}-\mathbf{k}_{\text {scatt }}^{\beta}=\boldsymbol{\kappa}^{\alpha}-\boldsymbol{\kappa}^{\beta}, \\
|\boldsymbol{\epsilon}| & =2 k \sin (\delta / 2), \\
\boldsymbol{\kappa}_{\text {ave }} & =\left(\boldsymbol{\kappa}^{\alpha}+\boldsymbol{\kappa}^{\beta}\right) / 2
\end{aligned}
$$

where $\mathbf{k}_{\text {scatt }}^{\alpha, \beta}$ is the scattered wave vector from the origin to detector $\alpha$ or $\beta$ and $\boldsymbol{\kappa}^{\alpha, \beta}$ is the scattered momentum transfer to $\alpha$ or $\beta$. The first term of approximation (27) is the single-particle contribution. The second term is the particle-pair contribution where $g\left(r_{j m}\right)$ is the static pair-correlation function. The third term represents diffraction from the collection of particles taken as a whole. As we are not concerned with forward scattering, we ignore the diffraction term. Similarly, we limit our treatment to volume fractions $\phi \lesssim 0.1$ and do not consider critical opalescence near phase transitions. ${ }^{7}$ In this case, the static pair-correlation function is ${ }^{34} g\left(r_{j m}\right) \approx 1$ for $r_{j m} \geq 2 a$, and we ignore pair-correlation effects. The remaining single-particle contribution to $\left[c_{1}(\tau)\right]$ may be written as

$$
\left[c_{1}(\tau)\right] \approx \frac{E_{0}{ }^{2} A_{1}{ }^{2} N_{0}}{k^{2} R_{d}{ }^{2}} \exp (i \omega \tau) U_{\text {auto }} G_{0}(\boldsymbol{\epsilon})\left[P_{1}^{2}\right] S\left(\boldsymbol{\kappa}^{\alpha}, \tau\right) .
$$

The autocorrelation strength factor,

$U_{\text {auto }} \equiv \frac{1}{V_{0}} \int_{V_{0}} \mathrm{~d}^{3} r_{j}\left|B\left(\mathbf{r}_{j}\right) D\left(\mathbf{r}_{j}\right)\right|^{2} \exp \left[-\left(L_{b j}+L_{j d}\right) / L_{\text {scatt }}\right]$,

contains both the roll-off of the transverse beam profile and variations in detector sensitivity in $V_{0}$. The dimensionless geometric factor,

$$
\begin{aligned}
G_{0}(\boldsymbol{\epsilon}) \equiv & \frac{1}{U_{\text {auto }} V_{0}} \int_{V_{0}} \mathrm{~d}^{3} r_{j}\left|B\left(\mathbf{r}_{j}\right) D\left(\mathbf{r}_{j}\right)\right|^{2} \\
& \times \exp \left[-\left(L_{b j}+L_{j d}\right) / L_{\text {scatt }}\right] \exp \left(i \boldsymbol{\epsilon} \cdot \mathbf{r}_{j}\right),
\end{aligned}
$$

is normalized to unity at $\epsilon=0$, which corresponds to autocorrelation.

To appreciate the significance of the geometric factor of Eq. (31), we consider $V_{0}$ to be a cylinder of radius $R_{y}$ and length $2 R_{z}$ oriented along the $z$ axis, as in Fig. 2 , which crudely models a focused laser beam in the vicinity of its focal waist. The length $2 R_{z}$ is the smaller of either the diameter of the scattering cell or the diameter of the detector field of view. For simplicity in our analysis, we take $B(\mathbf{r})$ to be a hard-edge box function. The detector field of view is also assumed to be uniform, although our results remain valid for all realistic beam and detector sensitivity profiles (e.g., Gaussians ${ }^{20}$ ). If the suspended particles are small, if they are approximately index matched to the liquid, or if their volume fraction is small, we can ignore the beam attenuation factors and obtain $U_{\text {auto }}=1$. Under these assumptions, Eq. (31) becomes the Fourier transform of $V_{0}$, i.e.,

$$
G_{0}(\boldsymbol{\epsilon})=\left[\frac{\sin \left(\epsilon R_{z} \cos \xi\right)}{\epsilon R_{z} \cos \xi}\right]\left[\frac{2 J_{1}\left(\epsilon R_{y} \sin \xi\right)}{\epsilon R_{y} \sin \xi}\right],
$$

where $\epsilon$ in general lies in the $y z$ plane and makes an angle $\xi$ with the $z$ axis. Our neglect of the beam attenuation factors is justified for only rather dilute suspensions because $R_{z}=5 \mathrm{~mm}$ for the experiment of Ref. 27 and $L_{\text {scatt }} \geq 3 \mathrm{~mm}$ for $\phi \leq 10^{-3}$. 
Equation (32) may be rewritten as

$$
G_{0}(\boldsymbol{\epsilon})=\left[\frac{\sin \left(\delta \cos \xi / \delta_{\mathrm{coh}^{z}}\right)}{\delta \cos \xi / \delta_{\mathrm{coh}^{z}}^{z}}\right]\left[\frac{2 J_{1}\left(\delta \sin \xi / \delta_{\mathrm{coh}^{y}}\right)}{\delta \sin \xi / \delta_{\mathrm{coh}^{y}}^{y}}\right],
$$

where $\delta_{\operatorname{coh}^{y}}^{y}$ and $\delta_{\text {coh }}^{z}$ have the following meanings. If the single-scattering volume is replaced by an incoherent light source with the same dimensions, the spatial coherence area of the source far down the $x$ axis has the angular diameter ${ }^{35}$

$$
\delta_{\text {coh }^{y}}=\frac{1}{k R_{y}}
$$

in the $y$ direction and

$$
\delta_{\mathrm{coh}^{z}}=\frac{1}{k R_{z}}
$$

in the $z$ direction. Thus light singly scattered from within $V_{0}$ is strongly cross correlated $\left\{\right.$ i.e., $G_{0}(\boldsymbol{\epsilon})$ and $\left[c_{1}(\tau)\right]$ are large $\}$ if detectors $\alpha$ and $\beta$ lie within the coherence area of the analogous light source. In Section 6 we find a similar geometric factor with a similar interpretation in the double-scattering contribution to the cross-correlation function.

To this point we have tacitly assumed that detectors $\alpha$ and $\beta$ are immersed in the liquid along with the suspended particles so that the ray paths from the scattering sites to the detectors are straight lines. Because the detectors in Fig. 2 lie outside the indexmatching vat, the ray paths refract at the vat-air interface, thus changing the dimensions of the spatial coherence area at the detector plane. Because the scattered rays in the $x y$ plane encounter a flat vat-air interface, a ray-tracing calculation gives

$$
\delta_{\operatorname{coh}^{y}}=\frac{\lambda}{2 \pi R_{y}}\left[1-\frac{\left(n_{L}-1\right) R_{v}}{n_{L} R_{d}}\right],
$$

where $R_{v}$ is the radius of the vat. The last factor in Eq. (36) is due to refraction, while the fact that only a portion of the ray path is in the liquid removes the implicit $n_{L}$ factor from $k$ in the denominator of Eq. (34). Because the scattered rays in the $x z$ plane encounter a curved vat-air interface, a similar raytracing calculation gives

$$
\delta_{\mathrm{coh}}^{z}=\frac{\lambda}{2 \pi n_{L} R_{z}}
$$

which is identical to Eq. (35). Refraction at the curved interface introduces a new $1 / n_{L}$ factor, while the fact that only a portion of the ray path is in the liquid removes the implicit $1 / n_{L}$ factor from $k$ in Eq. (35).

By a derivation similar to that which led to approximation (29), the single-scattering contribution to the second electric-field cross-correlation function $\left[c^{\prime}(\tau)\right]$ of Eq. (26) is

$$
\begin{aligned}
{\left[c_{1}{ }^{\prime}(\tau)\right] \approx } & \left\langle\left[E_{\alpha 1}(\tau) E_{\beta 1}(0)\right]\right\rangle \\
\approx & \frac{E_{0}^{2} A_{1}^{2} N_{0}}{k^{2} R_{d}^{2}} \exp \left(2 i k R_{d}-i \omega \tau\right)\left[P_{1}^{2}\right] \\
& \times S^{*}\left(\boldsymbol{\kappa}_{\text {ave }}, \tau\right) U_{\text {auto }} G_{0} *\left(2 \kappa_{\text {ave }}\right) .
\end{aligned}
$$

The geometric factor $G_{0} *\left(2 \kappa_{\text {ave }}\right)$ greatly suppresses $\left[c_{1}{ }^{\prime}(\tau)\right]$ with respect to $\left[c_{1}(\tau)\right]$ for the one-beam crosscorrelation geometry. For the cylindrical scattering volume of Fig. 2 and $\theta=90^{\circ}, \lambda=0.5145 \mu \mathrm{m}$, and $R_{y}$ $=44 \mu \mathrm{m}$, which correspond to the experimental parameters of Ref. 27 , we obtain $G_{0} *\left(2 \kappa_{\text {ave }}\right) \approx 5 \times 10^{-5}$. If, on the other hand, detectors $\alpha$ and $\beta$ are placed sufficiently close together, we have $G_{0}(\boldsymbol{\epsilon}) \approx 1$ in $\left[c_{1}(\tau)\right]$. A similar level of suppression occurs for double scattering as well, and we no longer consider the electric-field cross-correlation function $\left[c^{\prime}(\tau)\right]$ for the one-beam cross-correlation geometry.

For the double-scattering ensemble average, again we both ignore diffraction in the forward direction and assume that the particles have a sufficiently low volume fraction so that static pair-correlation effects are unimportant. We then obtain

$$
\begin{aligned}
{\left[c_{2}(\tau)\right] \approx } & \frac{E_{0}{ }^{2} A_{2}{ }^{2} \rho^{2}}{k^{2}{R_{d}}^{2}} \exp (i \omega \tau) \int_{V_{1}} \mathrm{~d}^{3} r_{j}\left|B\left(\mathbf{r}_{j}\right)\right|^{2} \\
& \times \exp \left(i \boldsymbol{\epsilon} \cdot \mathbf{r}_{j}\right) \int_{V_{2}} \mathrm{~d}^{3} r_{j l} \frac{\left|D\left(\mathbf{r}_{l}\right)\right|^{2}}{k^{2} r_{j l}{ }^{2}}\left[P_{2}{ }^{2}\right] \\
& \times S\left(\mathbf{k}_{\text {scatt }}{ }^{\alpha}-\mathbf{k}_{j l}, \tau\right) S\left(\mathbf{k}_{j l}-\mathbf{k}_{\text {inc }}, \tau\right) \\
& \times \exp \left[-\left(L_{b j}+r_{j l}+L_{l d}\right) / L_{\text {scatt }}\right) \exp \left(i \boldsymbol{\epsilon} \cdot \mathbf{r}_{j l}\right) .
\end{aligned}
$$

Similarly, for the single-double-scattering cross terms we obtain

$$
\begin{aligned}
{\left[c_{12}(\tau)\right] \approx } & \left\langle\left[E_{\alpha 1} *(\tau) E_{\beta 2}(0)\right]\right\rangle \\
\approx & \frac{E_{0}{ }^{2} A_{1} A_{2} \rho^{2}}{k^{2} R_{d}{ }^{2}} \exp (i \omega \tau) S\left(\boldsymbol{\kappa}^{\alpha}, \tau\right) \int_{V_{0}} \mathrm{~d}^{3} r_{j}\left|B\left(\mathbf{r}_{j}\right)\right|^{2} \\
& \times D^{*}\left(\mathbf{r}_{j}\right) \exp \left(i \boldsymbol{\epsilon} \cdot \mathbf{r}_{j}\right) \int_{V_{2}} \mathrm{~d}^{3} r_{j l} \frac{D\left(\mathbf{r}_{l}\right)}{k r_{j l}}\left[P_{1} P_{2}\right] \\
& \times \exp \left[-\left(2 L_{b j}+L_{j d}+r_{j l}+L_{l d}\right) / 2 L_{\text {scatt }}\right] \\
& \times \exp \left[i\left(\mathbf{k}_{j l}-\mathbf{k}_{\mathrm{scatt}}{ }^{\beta}\right) \cdot \mathbf{r}_{j l}\right] \\
{\left[c_{21}(\tau)\right] \approx } & \left\langle\left[E_{\alpha 2}{ }^{*}(\tau) E_{\beta 1}(0)\right]\right\rangle \\
\approx & \frac{E_{0}{ }^{2} A_{1} A_{2} \rho^{2}}{k^{2} R_{d}{ }^{2}} \exp (i \omega \tau) \int_{V_{0}} \mathrm{~d}^{3} r_{j} \mid B\left(\mathbf{r}_{j}\right)^{2} \\
& \times D^{*}\left(\mathbf{r}_{j}\right) \exp \left(i \boldsymbol{\epsilon} \cdot \mathbf{r}_{j}\right) \int_{V_{2}} \mathrm{~d}^{3} r_{j l} \frac{D\left(\mathbf{r}_{l}\right)}{k r_{j l}}\left[P_{1} P_{2}\right] \\
& \times S\left(\mathbf{k}_{\mathrm{scatt}}{ }^{\alpha}-\mathbf{k}_{j l}, \tau\right) S\left(\mathbf{k}_{j l}-\mathbf{k}_{\mathrm{inc}}, \tau\right) \\
& \times \exp \left[-\left(2 L_{b j}+L_{j d}+r_{j l}+L_{l d}\right) / 2 L_{\text {scatt }}\right] \\
& \times \exp \left[-i\left(\mathbf{k}_{j l}-\mathbf{k}_{\mathrm{scatt}}{ }^{\alpha}\right) \cdot \mathbf{r}_{j l}\right] .
\end{aligned}
$$


In approximations (40) and (41), the first of the two scatterings in $E_{\beta 2}$ or $E_{\alpha 2}{ }^{*}$ occurs in volume $V_{0}$ rather than $V_{1}$ because the particle involved also contributes to the single-scattered electric field $E_{\alpha 1}{ }^{*}$ or $E_{\beta 1}$, respectively. The single-triple-scattering cross-term contributions to the electric-field correlation function are of the same order ${ }^{36}$ in $A_{1}$ as is the doublescattering contribution of approximation (39). These cross terms are not considered here.

\section{Intensity Correlation Function}

In this section we consider the intensity crosscorrelation function

$$
[C(\tau)]=\int_{-\infty}^{\infty} \mathrm{d} T\left[I_{\alpha}(T+\tau) I_{\beta}(T)\right],
$$

which is constructed from experimental measurements and is theoretically modeled by the ensemble average,

$$
[C(\tau)] \approx\left\langle\left[E_{\alpha} *(\tau) E_{\alpha}(\tau) E_{\beta} *(0) E_{\beta}(0)\right]\right\rangle,
$$

where each electric field contains both singlescattering and double-scattering contributions. By using Wick's theorem to expand approximation (43) in products of electric-field cross-correlation functions, ${ }^{17}$ we obtain

$$
[C(\tau)]=\left[I_{\alpha}^{\text {ave }} I_{\beta}{ }^{\text {ave }}\right]+\left[Y^{*} Y\right]+\left[W^{*} W\right]
$$

where

$$
\begin{aligned}
{[Y]=} & \left\langle\left[E_{\alpha 1}(\tau) E_{\beta 1}(0)\right]\right\rangle+\left\langle\left[E_{\alpha 1}(\tau) E_{\beta 2}(0)\right]\right\rangle \\
& +\left\langle\left[E_{\alpha 2}(\tau) E_{\beta 1}(0)\right]\right\rangle+\left\langle\left[E_{\alpha 2}(\tau) E_{\beta 2}(0)\right]\right\rangle, \\
{[W]=} & \left\langle\left[E_{\alpha 1} *(\tau) E_{\beta 1}(0)\right]\right\rangle+\left\langle\left[E_{\alpha 1} *(\tau) E_{\beta 2}(0)\right]\right\rangle \\
& +\left\langle\left[E_{\alpha 2} *(\tau) E_{\beta 1}(0)\right]\right\rangle+\left\langle\left[E_{\alpha 2} *(\tau) E_{\beta 2}(0)\right]\right\rangle,
\end{aligned}
$$

The $\left[I_{\alpha}{ }^{\text {ave }} I_{\beta}{ }^{\text {ave }}\right]$ term in Eq. (44) is the baseline of the intensity cross-correlation function, and the time dependence is contained in the $\left[Y^{*} Y\right]$ and $\left[W^{*} W\right]$ terms. As was seen in Section 5, we neglect the $\left[Y^{*} Y\right]$ term with respect to $\left[W^{*} W\right]$. Because both single and multiple scattering contribute to $I_{\alpha}{ }^{\text {ave }}$ and $I_{\beta}{ }^{\text {ave }}$ whereas only single scattering is found in this section to contribute to $\left[W^{*} W\right]$, the signal-tobaseline value for cross correlation is expected to be smaller than that for autocorrelation, in which both single and multiple scattering contribute, albeit with different time dependences, to $\left[W^{*} W\right]$.

For previous autocorrelation and crosscorrelation geometries that used collimated detectors, some of the integrations over particle coordinates in the double-scattering contribution to the autocorrelation function could not be performed analytically. ${ }^{5}$ We now assume that each particle $j$ within the beam and the detector field of view (except those near the walls of the scattering cell) is surrounded by an isotropic environment of other particles $l$, also in the detector field of view, out to some average radius $r_{j l} \approx R_{\text {ave }}$, which is comparable with $R_{z}$. As was mentioned in Ref. 37, the isotropic environment assumption is appropriate for a narrow beam and wide-field-of-view detectors. In this section it is shown that this assumption, although only approximately true for the geometry of Fig. 2, allows us to calculate analytically the doublescattering contribution to the autocorrelation and cross-correlation functions.

The isotropic environment assumption, the modeling of $B(\mathbf{r})$ and $D(\mathbf{r})$ by hard-edge box functions, and the neglect of the attenuation factors approximately decouple the $\mathrm{d}^{3} r_{j}$ and $\mathrm{d}^{3} r_{j l}$ integrations for the one-beam cross-correlation geometry. The $\mathrm{d}^{3} r_{j}$ integral in approximations (39)-(41) is identical to the single-scattering case. The double-scattering electric-field cross-correlation function of approximation (39) for noninteracting particles can then be written as

$\left[c_{2}(\tau)\right]=\frac{E_{0}{ }^{2} A_{1}{ }^{2} N_{0}}{k^{2} R_{d}{ }^{2}} \exp (i \omega \tau) Q_{2} U_{\text {auto }} G_{0}(\boldsymbol{\epsilon})\left[S_{2}(\theta, \tau, \boldsymbol{\epsilon})\right]$,

where

$$
Q_{2}=3 \phi\left(\frac{n^{2}-1}{n^{2}+2}\right)^{2}(k a)^{3}\left(k R_{\text {ave }}\right),
$$

and the remaining integrations over particle coordinates are contained in

$$
\begin{aligned}
{\left[S_{2}(\theta, \tau, \boldsymbol{\epsilon})\right] \equiv } & {\left[\begin{array}{ll}
S_{2}{ }^{V V} & S_{2}{ }^{V H} \\
S_{2}{ }^{H V} & S_{2}{ }^{H H}
\end{array}\right] } \\
= & \frac{1}{4 \pi R_{\text {ave }}} \int_{0}^{R_{\text {ave }}} \mathrm{d} r_{j l} \int_{0}^{\pi} \sin \theta_{j l} \mathrm{~d} \theta_{j l} \int_{0}^{2 \pi} \mathrm{d} \phi_{j l}\left[P_{2}{ }^{2}\right] \\
& \times \exp \left(-\left|\mathbf{k}_{\text {scatt }}-\mathbf{k}_{j l}\right|^{2} D \tau\right) \\
& \times \exp \left(-\left|\mathbf{k}_{j l}-\mathbf{k}_{\text {inc }}\right|^{2} D \tau\right) \exp \left(i \boldsymbol{\epsilon} \cdot \mathbf{r}_{j l}\right),
\end{aligned}
$$

where $D$ is the diffusion coefficient of the particles in the liquid-filled scattering cell. Equation (47) differs from the single-scattering electric-field crosscorrelation function of approximation (29) by the replacement of $\left[P_{1}^{2}\right] S$ by $Q_{2}\left[S_{2}\right]$.

The integrations in Eq. (49) may be performed analytically in the $\epsilon=0, \epsilon R_{\text {ave }} \gg 1$, and $\tau=0$ limits by use of a coordinate system rotated from the coordinate system of Fig. 4 by an angle $\gamma \equiv \theta / 2$ about the $y$ axis. The result for $\epsilon=0$ (i.e., autocorrelation) is

$$
\begin{aligned}
S_{2}{ }^{V V}(\tau)= & \exp \left(-4 k^{2} \mathrm{D} \tau\right)\left[\frac{3}{8} Z_{0}(b)+\frac{2}{8} Z_{1}(b)+\frac{3}{8} Z_{2}(b)\right], \\
S_{2}{ }^{V H}(\tau)= & S_{2}^{H V}(\tau)=\exp \left(-4 k^{2} D \tau\right)\left[\cos ^{2} \gamma Z_{0}(b)\right. \\
& -\left(6 \cos ^{2} \gamma-4\right) Z_{1}(b) \\
& \left.+\left(5 \cos ^{2} \gamma-4\right) Z_{2}(b)\right],
\end{aligned}
$$




$$
\begin{aligned}
S_{2}{ }^{H H}(\tau)= & \exp \left(-4 k^{2} D \tau\right)\left[\left(\frac{19}{8} \cos ^{4} \gamma-3 \cos ^{2} \gamma+1\right)\right. \\
& \times Z_{0}(b)-\left(\frac{14}{8} \cos ^{4} \gamma-4 \cos ^{2} \gamma+2\right) \\
& \left.\times Z_{1}(b)+\left(\frac{3}{8} \cos ^{4} \gamma-\cos ^{2} \gamma+1\right) Z_{2}(b)\right]
\end{aligned}
$$

where

$$
\begin{aligned}
b & =4 k^{2} D \tau \cos \gamma, \\
Z_{m}(b) & \equiv \frac{1}{2} \int_{-1}^{1} u^{2 m} \mathrm{~d} u \exp (b u)
\end{aligned}
$$

for $m=0,1,2$. The $Z_{m}(b)$ are combinations of hyperbolic sines and cosines divided by powers of $b$. These autocorrelation results merit the following comments. Equation (49) with $\epsilon=0$ was first considered in Ref. 2 in a coordinate system in which the incident beam propagated in the $-x$ direction, the detector was located in the $x y$ plane, and the integrations were performed numerically. The slope of Eqs. (50)-(52) at $\tau=0$ is seen here to be $-4 k^{2} D$ and is independent of $\theta$, a result first obtained in Ref. 2 . Similarly, the graph of Eq. (51) is identical to the graph of $S_{2}{ }^{V H}(\tau)$ shown in Fig. 2 of Ref. 2, which was obtained by means of numerical integration. The equality of $S_{2}{ }^{V H}(\tau)$ and $S_{2}{ }^{H V}(\tau)$ obtained here was first inferred on the basis of numerical integration in Ref. 3. The results of Eqs. (47)-(54) assume a point detector. For a finite-size detector, our results must be integrated with respect to $\epsilon$ over the detector face. ${ }^{23}$ For a 3 - $\mu$ m-diameter monomode optical fiber coupled to a detector, $\epsilon R_{\text {ave }}$ remains less than $\sim 1.0$ over the fiber cross section and the results of Eqs. (47)-(54) remain valid.

When $\epsilon$ is in the $y$ direction, as in Fig. 2 , and $\epsilon R_{\text {ave }}$ $\gg 1$ corresponds to the cross correlation of two closely spaced detectors (e.g., a $250-\mu \mathrm{m}$ detector spacing at $x=16 \mathrm{~cm}$, as in Ref. 27 , gives $\delta \approx 10^{-3}$ rad or $\epsilon R_{\text {ave }} \approx 100$ ), we have

$$
\begin{aligned}
S_{2}{ }^{W V}= & \frac{\pi}{2 \epsilon R_{\text {ave }}} \exp \left(-4 k^{2} D \tau\right) I_{0}(b), \\
S_{2}{ }^{V H}= & S_{2}{ }^{H V}=O\left[\left(\epsilon R_{\text {ave }}\right)^{-3}\right], \\
S_{2}{ }^{H H}= & \frac{\pi}{2 \epsilon R_{\text {ave }}} \exp \left(-4 k^{2} D \tau\right) \\
& \times\left[\cos ^{4} \gamma I_{0}(b)-\frac{2 \cos ^{2} \gamma}{b} I_{1}(b)+\frac{3}{b^{2}} I_{2}(b)\right],
\end{aligned}
$$

where $I_{m}(b)$ for $m=0,1,2$ are modified Bessel functions. These cross-correlation results merit the following observations. Whereas the single-scattering correlation function of approximation (29) is exponential in $\tau$ for noninteracting particles, the time dependence of $Z_{m}(b)$ for autocorrelation and $I_{m}(b)$ for cross correlation causes the double-scattering contribution to the electric-field correlation functions to be nonexponential in $\tau$ for $\theta \neq 180^{\circ}$. Also, although there is substantial double scattering in the $V H$ and the $H V$ polarization channels for autocorrelation, doublescattering cross correlation is exceedingly weak in these channels.

Again when $\epsilon$ is in the $y$ direction, calculation of the $\epsilon$ dependence of $\left[S_{2}\right]$ appears to be relatively simple in only the $\tau=0$ limit (i.e., the intensity ensemble average). In this limit we obtain

$$
\begin{aligned}
S_{2} V & =\frac{8}{\epsilon R_{\text {ave }}} \int_{0}^{\epsilon R_{\text {ave }}} \mathrm{d} u \frac{j_{2}(u)}{u^{2}}, \\
S_{2}{ }^{V H}= & S_{2}{ }^{H V}=\frac{1}{\epsilon R_{\text {ave }}} \int_{0}^{\epsilon R_{\text {ave }}} \mathrm{d} u\left[\frac{j_{1}(u)}{u}-\frac{4 j_{2}(u)}{u^{2}}\right], \\
S_{2}{ }^{H H}= & \frac{1}{\epsilon R_{\text {ave }}} \int_{0}^{\epsilon R_{\text {ave }}} \mathrm{d} u\left\{\cos ^{2} \theta\left[j_{0}(u)-\frac{2 j_{1}(u)}{u}\right]\right. \\
& \left.+\left(2 \cos ^{2} \theta+1\right) \frac{j_{2}(u)}{u}\right\},
\end{aligned}
$$

where $j_{m}(u)$ for $m=0,1,2$ are spherical Bessel functions. The $\epsilon=0$ and $\epsilon R_{\text {ave }} \gg 1$ limits of Eqs. (58)-(60) agree with Eqs. (50)-(52) and Eqs. (55)(57), respectively.

The single-double-scattering cross terms of approximations (40) and (41) may be written as

$$
\left[c_{12}(\tau)\right]=\frac{E_{0}{ }^{2} A_{1}^{2} N_{0}}{k^{2} R_{d}^{2}} \exp (i \omega \tau) Q_{12} U_{\text {auto }} G_{0}(\boldsymbol{\epsilon})\left[S_{12}(\theta, \tau)\right],
$$

where

$$
Q_{12}=\frac{1}{2}\left(\frac{n^{2}+2}{n^{2}-1}\right)(k a)^{-6}\left(k R_{\text {ave }}\right)^{-1} Q_{2}
$$

and the remaining integrations over particle coordinates are contained in

$$
\begin{aligned}
{\left[S_{12}(\theta, \tau)\right]=} & \frac{k^{2}}{2 \pi} S\left(\boldsymbol{\kappa}^{\alpha}, \tau\right) \int_{0}^{R_{\text {ave }}} r_{j l} \exp \left(i k r_{j l}\right) \mathrm{d} r_{j l} \\
& \times \int_{0}^{\pi} \sin \theta_{j l} \mathrm{~d} \theta_{j l} \int_{0}^{2 \pi} \mathrm{d} \phi_{j l}\left[P_{1} P_{2}\right] \\
& \times \exp \left(-i \mathbf{k}_{\text {scatt }}{ }^{\beta} \cdot \mathbf{r}_{j l}\right) .
\end{aligned}
$$

Similarly,

$$
\left[c_{21}(\tau)\right]=\frac{E_{0}{ }^{2} A_{1}{ }^{2} N_{0}}{k^{2} R_{d}{ }^{2}} \exp (i \omega \tau) Q_{12} U_{\text {auto }} G_{0}(\boldsymbol{\epsilon})\left[S_{21}(\theta, \tau)\right],
$$


where

$$
\begin{aligned}
{\left[S_{21}(\theta, \tau)\right]=} & \frac{k^{2}}{2 \pi} \int_{0}^{R_{\text {ave }}} r_{j l} \exp \left(-i k r_{j l}\right) \mathrm{d} r_{j l} \int_{0}^{\pi} \sin \theta_{j l} \mathrm{~d} \theta_{j l} \\
& \times \int_{0}^{2 \pi} \mathrm{d} \phi_{j l}\left[P_{1} P_{2}\right] \exp \left(-\left|\mathbf{k}_{\text {scatt }}-\mathbf{k}_{j l}\right|^{2} D \tau\right) \\
& \times \exp \left(-\left|\mathbf{k}_{j l}-\mathbf{k}_{\text {inc }}\right|^{2} \mathrm{D} \tau\right) \exp \left(i \mathbf{k}_{\text {scatt }}{ }^{\alpha} \cdot \mathbf{r}_{j l}\right) .
\end{aligned}
$$

The integration of Eqs. (63) and (65) shows that $\left[S_{12}\right]$ and $\left[S_{21}\right]$ are each large for $\epsilon R_{\text {ave }} \gg 1$. But they are opposite in sign and nearly equal in magnitude, and they almost completely cancel when added together in Eq. (46). The cancellation is maximal at $\tau=0$ and slowly decreases for $\tau>0$ because of the different combinations of structure factors in Eqs. (63) and (65). The fact that $Q_{12} \approx$ $10^{-4} Q_{2}$ for $n=1.2, k a=0.87$, and $R_{\text {ave }} \approx 4 \mathrm{~mm}$, as in Ref. 27, further decreases the importance of the cross terms for both autocorrelation and cross correlation. The cross terms have also been found to be unimportant for a number of other scattering geometries. $3,17,36,38,39$

The suppression of double scattering and, by inference, the suppression of all multiple scattering for a single focused laser beam propagating through the scattering cell and cross correlation of two closely spaced detectors, as in Refs. 27-29, can be demonstrated as follows. For $a=0.053 \mu \mathrm{m}$ polystyrene latex spheres in water ${ }^{27}$ with $n=1.2, \lambda=0.5145 \mu \mathrm{m}$, and $R_{\text {ave }} \approx 4 \mathrm{~mm}$, the product of the factors $Q_{2}\left[S_{2}\right]$ in Eq. (47) becomes larger than unity at $\tau=0$ for autocorrelation, i.e., the double-scattering contribution becomes larger than the single-scattering contribution for $\phi \geqslant 10^{-3}$. This is experimentally observed in Ref. 27. The time dependence of the autocorrelation function for noninteracting particles ceases being exponential at volume fractions of this order both because $\left[S_{2}\right]$ is nonexponential in $\tau$ and, perhaps more importantly, because the general rate of falloff of $\left[S_{2}\right]$ differs from that of $S$. On the other hand, the profile of the single-scattering spatial coherence area as a function of the detector angular separation $\delta$ in the four polarization channels is modeled by $G_{0}(\boldsymbol{\epsilon})\left[P_{1}{ }^{2}\right] S$ in Eqs. (28) and approximation (29). Similarly the profile of the double-scattering spatial coherence area as a function of $\delta$ in the four polarization channels is modeled by $G_{0}(\boldsymbol{\epsilon})\left[S_{2}(\theta, \tau, \boldsymbol{\epsilon})\right]$ in Eq. (47). The amount of double scattering for cross correlation with $\epsilon R_{\text {ave }} \gg 1$ relative to the amount of double scattering for autocorrelation with $\epsilon=0$ in the $V V$ polarization channel at $\tau=0$ is

$F^{V V}(\delta)=\frac{S_{2}^{V V}\left(\theta, \tau=0, \epsilon R_{\mathrm{ave}} \gg 1\right)}{S_{2}{ }^{V V}(\theta, \tau=0, \epsilon=0)}=\frac{4 \pi}{15 \epsilon R_{\mathrm{ave}}}$,
If detectors $\alpha$ and $\beta$ are placed so that $\delta \approx 1 / k R_{y}$, singly scattered light is strongly correlated and $F^{V V}(\delta)$ becomes

$$
F^{V V}(\delta) \approx \frac{4 \pi R_{y}}{15 R_{\text {ave }}} \approx 0.009
$$

for the values of $R_{y}$ and $R_{\text {ave }}$ in Ref. 27, thus reducing the double-scattering amplitude 100-fold from its autocorrelation value. This degree of suppression is comparable with the value calculated by Schätzel for the two-color cross-correlation system. ${ }^{20}$ The $\tau$ dependence of $F^{V V}(\delta)$ is obtained by the division of Eq. (55) by Eq. (50) but does not significantly affect the degree of suppression from the value given in approximation (67). As a result, the intensity crosscorrelation function of Eqs. (44) and (46) for the onebeam cross-correlation geometry is dominated by single scattering and remains exponential in $\tau$ for noninteracting particles at values of $\phi$ for which the autocorrelation function is no longer exponential.

Experimentally it is seen in Ref. 27 that multiplescattering suppression occurs for the geometry of Fig. 2 for $\phi \leq 0.1$, which is far beyond the range of validity of the assumptions leading to approximation (67). At high volume fractions, triple scattering and beyond become important. Particle $l$ is not necessarily in the far zone of particle $j$ so that the Lorenz-Mie near-zone scattering amplitude must be employed. Similarly, pair-correlation effects, particle interaction effects, and the beam attenuation factors cannot be ignored. Nonetheless, the experimentally observed multiplescattering suppression at large volume fractions attests to the robustness of the one-beam crosscorrelation technique.

\section{Conclusions}

The principal result is that for the one-beam crosscorrelation system, multiple scattering at low volume fractions occurs over a relatively large region in the $y$ direction, whereas single scattering occurs within only the focused laser beam. As a result, multiple scattering produces a smaller far-zone coherence length in the $y$ direction than does single scattering. If detectors $\alpha$ and $\beta$ lie on the same single-scattering coherence area but on different multiple-scattering coherence areas, single scattering should be strongly cross correlated but multiple scattering should not be. Although this result may be thought of as being intuitively obvious in retrospect, it is pleasing to see that it can be derived with standard light-scattering theory.

The development presented here also fills in various details that the intuitive spatial coherence area argument alone cannot provide, such as the polarization dependence of the autocorrelation and crosscorrelation results and the degree of spatial coherence as a function of $\delta$, which is of special interest in light of the experimental results of Ref. 29. Our analytical evaluation of the $\tau$ dependence of the double-scattering contribution to the autocorrelation function, although dependent on the isotropic envi- 
ronment assumption that is only approximately satisfied, is also of interest. This analytical result, if extendable to higher orders of multiple scattering, should provide a method for analyzing the experimental autocorrelation results of Ref. 40 that span the particle volume fraction range from the semidilute limit to the deep multiple-scattering regime. Previous multiple-scattering analyses of autocorrelation spectra assumed that each multiparticle-scattering contribution was an exponential with a different decay rate. ${ }^{6}$ Nonexponential contributions, such as those derived here for double scattering, should more realistically describe the experimental spectra.

The real assessment of the importance of the onebeam cross-correlation technique is the ease of its alignment, the rate at which and the level to which noise in the cross correlogram is decreased, and the simplicity of the analysis of the cross correlogram. These issues are addressed separately. ${ }^{27-29}$

\section{References}

1. N. A. Clark, J. H. Lunacek, and G. B. Benedek, "A study of Brownian motion using light scattering," Am. J. Phys. 38, 575-585 (1970).

2. C. M. Sorensen, R. C. Mockler, and W. J. O'Sullivan, "Depolarized correlation function of light double scattered from a system of Brownian particles," Phys. Rev. A 14, 1520-1532 (1976).

3. J. K. G. Dhont, "Multiple Rayleigh-Gans-Debye scattering in colloidal systems: dynamic light scattering," Physica 129A, 374-394 (1985).

4. J. K. G. Dhont, C. G. deKruif, and A. Vrij, "Light scattering in colloidal suspensions: effects of multiple scattering," J. Colloid Interface Sci. 105, 539-551 (1985).

5. J. G. Shanks and J. V. Sengers, "Double scattering in critically opalescent fluids," Phys. Rev. A 38, 885-896 (1988).

6. C. M. Sorensen, R. C. Mockler, and W. J. O’Sullivan, "Multiple scattering from a system of Brownian particles," Phys. Rev. A 17, 2030-2035 (1978).

7. A. E. Bailey and D. S. Cannell, "Practical method for calculation of multiple light scattering," Phys. Rev. E 50, 4853-4864 (1994).

8. D. J. Pine, D. A. Weitz, P. M. Chaikin, and E. Herbolzheimer, "Diffusing-wave spectroscopy," Phys. Rev. Lett. 60, 1134-1137 (1988).

9. F. C. MacKintosh and S. John, "Diffusing-wave spectroscopy and multiple scattering of light in correlated random media," Phys. Rev. B 40, 2383-2406 (1989).

10. P. D. Kaplan, M. H. Kao, A. G. Yodh, and D. J. Pine, "Geometric constraints for the design of diffusing-wave spectroscopy experiments," Appl. Opt. 32, 3828-3836 (1993).

11. D. A. Weitz and D. J. Pine, "Diffusing-wave spectroscopy," in Dynamic Light Scattering: The Method and Some Applications, W. Brown, ed. (Clarendon, Oxford, 1993), pp. 652-720.

12. B. J. Ackerson, R. L. Dougherty, N. M. Reguigui, and U. Nobbmann, "Correlation transfer: application of radiative transfer solution methods to photon correlation problems," J. Thermophys. Heat Transfer 6, 577-588 (1992).

13. R. L. Dougherty, B. J. Ackerson, N. M. Reguigui, F. DorriNowkoorani, and U. Nobbmann, "Correlation transfer: development and application,” J. Quant. Spectrosc. Radiat. Transfer 52, 713-727 (1994).

14. G. D. J. Phillies, "Suppression of multiple scattering effects in quasielastic light scattering by homodyne cross-correlation techniques," J. Chem. Phys. 74, 260-262 (1981).

15. G. D. J. Phillies, "Experimental demonstration of multiplescattering suppression in quasielastic light scattering spectroscopy by homodyne coincidence techniques," Phys. Rev. A 24, 1939-1943 (1981).

16. H. J. Mos, C. Pathmamanoharan, J. K. G. Dhont, and C. G. de Kruif, "Scattered light intensity cross correlation. II. Experimental,” J. Chem. Phys. 84, 45-49 (1986).

17. J. K. G. Dhont and C. G. de Kruif, "Scattered light intensity cross-correlation. I. Theory," J. Chem. Phys. 79, 1658-1663 (1983).

18. M. Drewel, J. Ahrens, and U. Podschus, "Decorrelation of multiple scattering for an arbitrary scattering angle," J. Opt. Soc. Am. A 7, 206-210 (1990).

19. K. Schätzel, M. Drewel, and J. Ahrens, "Suppression of multiple scattering in photon correlation spectroscopy," J. Phys.: Condensed Matter 2, SA393-SA398 (1990).

20. K. Schätzel, "Suppression of multiple scattering by photon cross-correlation techniques,” J. Mod. Opt. 38, 1849-1865 (1991).

21. R. G. Brown, "Dynamic light scattering using monomode optical fibers," Appl. Opt. 26, 4846-4851 (1987).

22. H. S. Dhadwal and B. Chu, "A fiber-optic light-scattering spectrometer," Rev. Sci. Instrum. 50, 845-853 (1989).

23. J. Ricka, "Dynamic light scattering with single-mode and multimode receivers," Appl. Opt. 32, 2860-2875 (1993).

24. Superano, K. Deurloo, P. Stamatelopolous, R. Srivastva, and J. C. Thomas, "Light scattering with single-mode fiber collimators," Appl. Opt. 33, 7200-7205 (1994).

25. T. Gisler, H. Ruger, S. U. Egelhaaf, J. Tschumi, P. Schurtenberger, and J. Ricka, "Mode-selective dynamic light scattering: theory versus experimental realization," Appl. Opt. 34, 35463553 (1995).

26. H. Wiese and D. Horn, "Single-mode fibers in fiber-optic quasielastic light scattering: a study of the dynamics of concentrated latex dispersions," J. Chem. Phys. 94, 6429-6443 (1991).

27. W. V. Meyer, D. S. Cannell, A. E. Smart, T. W. Taylor, and P. Tin, "Suppression of multiple scatterring using a single beam cross-correlation method, in Light Scattering and Photon Correlation Spectroscopy, E. R. Pike and J. B. Abbiss, eds., NATO ASI Series (Kluwer, Dordrecht, The Netherlands, 1997).

28. W. V. Meyer, D. S. Cannell, A. E. Smart, T. W. Taylor, and P. Tin "Multiple-scattering suppression by cross correlation," Appl. Opt. 36, 7551-7558 (1997).

29. U. Nobbmann, S. W. Jones, and B. J. Ackerson, "Multiplescattering suppression: cross correlation with tilted singlemode fibers," Appl. Opt. 36, 7571-7576 (1997).

30. J. A. Lock, "Theory of multiple scattering suppression in crosscorrelated light scattering employing a single laser beam," in Light Scattering and Photon Correlation Spectroscopy, E. R. Pike and J. B. Abbiss, eds., NATO ASI Series (Kluwer, Dordrecht, The Netherlands, 1997).

31. H. C. van de Hulst, Light Scattering by Small Particles (Dover, New York, 1981), pp. 127-128.

32. Ref. 31, p. 70.

33. R. W. Hart and R. A. Farrell, "Light scattering in the cornea," J. Opt. Soc. Am. 59, 766-774 (1969).

34. P. M. Chaikin and T. C. Lubensky, Principles of Condensed Matter Physics (Cambridge U. Press, Cambridge, 1995), Fig. 2.4.1.

35. E. Hecht, Optics, 2nd ed. (Addison-Wesley, Reading, Mass., 1987), p. 532.

36. H. M. J. Boots, D. Bedeaux, and P. Mazur, "On the theory of multiple scattering. I.," Physica 79A, 397-419 (1975). 
37. C. M. Sorensen, R. C. Mockler, and W. J. O'Sullivan, "Autocorrelation spectroscopy studies of single and multiple scattered light from a critical mixture," Phys. Rev. A 16, 365-376 (1977).

38. R. A. Farrell and J. K. Bhattacharjee, "Double-scattering correction for the critical dynamics of a classical fluid," Phys. Rev.
A 19, 348-369 (1979)

39. D. W. Oxtoby and W. M. Gelbart, "Double-scattering-induced deviations from Ornstein-Zernike behavior near the critical point," Phys. Rev. A 10, 738-740 (1974).

40. P. Stepanek, "Static and dynamic properties of multiple light scattering,” J. Chem. Phys. 99, 6384-6393 (1993). 